\title{
Infective endocarditis with multiple mycotic aneurysms mimicking vasculitis: A case report
}

\author{
Ji Hyeon Park MD ${ }^{1}$, Hye Ryoun Jang PhD MD², Jung Eun Lee PhD MD², Wooseong Huh PhD MD², \\ Dae Joong Kim PhD MD², Ha Young Oh PhD MD², Yoon-Goo Kim PhD MD²
}

JH Park, HR Jang, JE Lee, et al. Infective endocarditis with multiple mycotic aneurysms mimicking vasculitis: A case report. Can J Infect Dis Med Microbiol 2012;23(3):e67-68.

The manifestation of infective endocarditis often resembles vasculitis. Approximately one in five infective endocarditis cases are referred initially to a nephrologist because of abnormal renal function or abnormal urinalysis; therefore, infection should be ruled out before diagnosing vasculitis.

A case involving a patient with infective endocarditis who presented with migrating skin lesions, renal infarction and multiple pseudoaneurysms is reported. Echocardiography revealed mitral valve vegetation and viridans streptococci were identified in peripheral blood cultures. Although mitral valve annuloplasty and an aneurysm ligation operation were performed with proper antibiotic treatment, the remaining mycotic aneurysm progressed and caused neurological complications. The patient was cured completely after reoperation.

Key Words: Aneurysm; Endocarditis; Infected; Vasculitis

\section{Une endocardite infectieuse accompagnée de multiples anévrismes mycotiques imitant une vasculite : un rapport de cas}

L'endocardite infectieuse se manifeste souvent comme une vasculite. Environ un cas d'endocardite infectieuse sur cinq est d'abord aiguillé vers un néphrologue en raison d'une fonction rénale ou d'une urinalyse anormale. Ainsi, il faut écarter la possibilité d'infection avant de diagnostiquer une vasculite.

Les chercheurs présentent le cas d'un patient atteint d'endocardite infectieuse qui a consulté en raison de la migration de lésions cutanées, d'un infarctus rénal et de multiples pseudoanévrismes. L'échocardiographie a révélé une végétation de la valvule mitrale et des streptocoques viridans dans les prélèvements sanguins périphériques. Malgré une annuloplastie de la valvule mitrale, une ligature de l'anévrisme et une antibiothérapie pertinente, l'anévrisme mycotique restant a évolué et causé des complications neurologiques. Le patient a guéri complètement après une deuxième opération.
Tnfective endocarditis often presents with insidious-onset, constitutional symptoms including myalgia, weight loss and slowly progressive anemia, and is frequently accompanied by cutaneous eruptions (1). Renal complications of infective endocarditis include renal infarction and glomerulonephritis (2), although these presentations are not specific for infective endocarditis and are found frequently in vasculitis patients. This clinical similarity makes the differential diagnosis difficult; therefore, it is important to suspect underlying infectious disease when treating patients presenting with symptoms and signs of vasculitis.

We report a case of bacterial endocarditis presenting with migrating skin lesions, multiple pseudoaneurysms and renal infarction.

\section{CASE PRESENTATION}

A previously healthy, 25-year-old Korean man presented with weight loss ( $6 \mathrm{~kg}$ over three months) and migrating erythematous skin lesions on his hands and feet. He visited a rheumatologist and a skin biopsy was performed, which showed fat necrosis. His skin lesions increased and decreased in severity. After two months, he noticed a pulsatile abdominal mass and presented to another hospital. An abdominopelvic computed tomography (CT) scan showed aneurysms in the superior mesenteric artery and the left internal iliac artery, and a left renal infarction (Figure 1). He was referred to the nephrology division of Samsung Medical Center (Seoul, Korea).

The patient was admitted to the nephrology division for further evaluation. He complained of being intermittently febrile, although he was afebrile at admission. Positive physical findings included a systolic murmur and a pulsatile abdominal mass with tenderness. Laboratory test results showed a white blood cell count of $13.070 \times 10^{9} / \mathrm{L}$ (0.73/1.0 neutrophils), a hemoglobin concentration of $125 \mathrm{~g} / \mathrm{L}$, an erythrocyte sediment rate of $74 \mathrm{~mm} / \mathrm{h}$, and a C-reactive protein concentration of $892.4 \mathrm{nmol} / \mathrm{L}$. Blood chemistry test results showed the serum concentrations of albumin, blood urea nitrogen and creatinine to be $621 \mu \mathrm{mol} / \mathrm{L}, 4.6 \mathrm{mmol} / \mathrm{L}$ and $75 \mu \mathrm{mol} / \mathrm{L}$, respectively. Urinalysis revealed microscopic hematuria and microalbuminuria. The urinary protein:creatinine ratio was $29.4 \mathrm{mg} / \mathrm{mmol}$, and the albumin:creatinine ratio was $12.2 \mathrm{mg} / \mathrm{mmol}$. Antinuclear antibody and antineutrophil cytoplasmic antibody tests were negative, and C3 and C4 levels were within normal limits. Transthoracic echocardiography and transesophageal echocardiography revealed a $1.42 \mathrm{~cm} \times$ $0.60 \mathrm{~cm}$ oscillating mass on the mitral valve.

On hospital day 3, fever was detected, with an elevated temperature of $39.1^{\circ} \mathrm{C}$. Two sets of blood cultures were performed, and empirical antibiotic treatment with intravenous ceftriaxone $2 \mathrm{~g}$ per day, ampicillin $2 \mathrm{~g}$ every $4 \mathrm{~h}$ and gentamicin $1 \mathrm{mg} / \mathrm{kg}$ every $8 \mathrm{~h}$ was started. On hospital day 5, viridans streptococci were identified from the initial blood culture, and empirical antibiotics were switched to intravenous penicillin $\mathrm{G}, 3 \times 10^{6} \mathrm{IU}$ every $6 \mathrm{~h}$. Simultaneously, mitral valve annuloplasty was performed to remove the vegetation. On hospital day 15 , superior mesenteric artery aneurysm ligation and left internal iliac artery aneurysm thrombectomy were performed.

On hospital day 25, left foot drop and left lateral thigh pain suddenly developed. A nerve conduction study revealed a left common peroneal nerve conduction disorder. Rehabilitation was performed, but the symptoms were not improved. On hospital day 28, sudden right buttock pain and tenderness developed. Pelvic magnetic resonance imaging was performed to evaluate the right buttock mass; a right buttock hematoma and multiple aneurysmal dilations in the left external iliac and internal iliac chains were detected (Figure 2A). Antibiotic treatment for infective endocarditis was continued. On hospital day 48, when the proper antibiotic treatment had been given for six weeks, a follow-up CT scan showed multiple left internal iliac artery aneurysms with impending

${ }^{1}$ Department of Medicine; ${ }^{2}$ Division of Nephrology, Department of Medicine, Samsung Medical Center, Sungkyunkwan University School of Medicine, Seoul, Korea

Correspondence and reprints: Dr Yoon-Goo Kim, Division of Nephrology, Department of Medicine, Samsung Medical Center, Sungkyunkwan University School of Medicine, 50 Irwon-dong, Gangnam-gu, 135-710, Seoul, Korea. Telephone 82-2-3410-3442, fax 82-2-3410-0067, e-mail ygkim26@skku.edu 


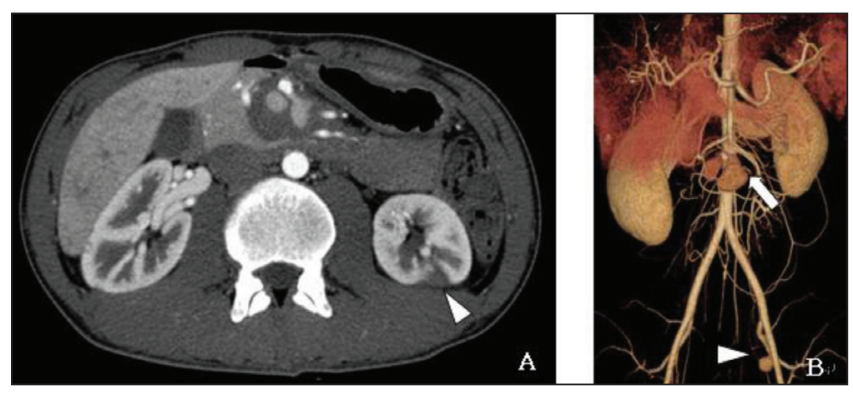

Figure 1) A Coronal computed tomography (CT) image showing left renal infarction (arrowhead) and decreased volume in the lower pole. B Three-dimensional reconstructed CT image shows a $48 \mathrm{~mm} \times 36 \mathrm{~mm}$ pseudoaneurysm in the superior mesenteric artery (arrow) and a $20 \mathrm{~mm}$ pseudoaneurysm in the internal iliac artery (arrowhead)
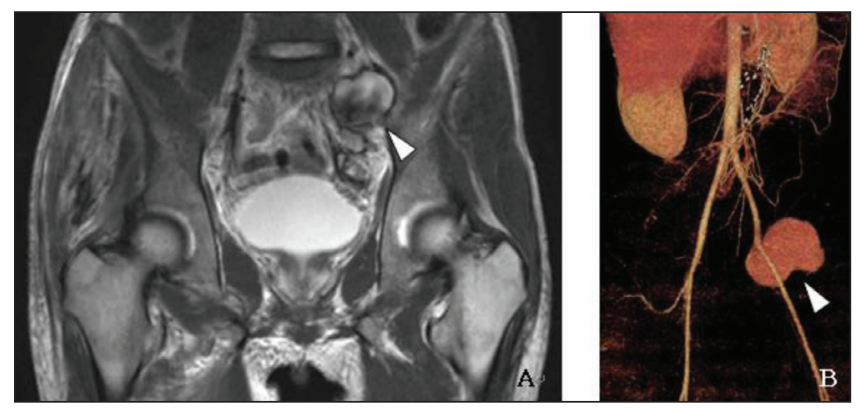

Figure 2) A Coronal T2-weighted magnetic resonance image showing multiple left internal iliac artery aneurysmal dilations (arrowhead). B Three-dimensonal reconstructed computed tomography image showing a $30 \mathrm{~mm} \times 70 \mathrm{~mm}$ aneurysm in the left internal iliac artery (arrowhead) with impending rupture
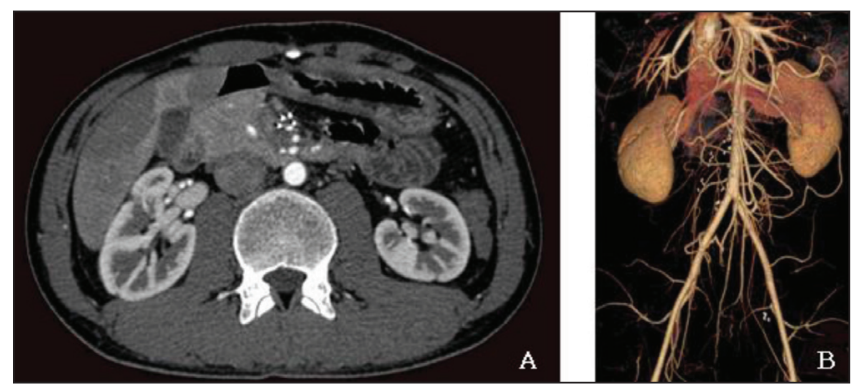

Figure 3 ) Coronal computed tomography image (A) and three-dimensional reconstructed computed tomography (B) showing scarring caused by the left kidney infarction and the absence of aneurysm

rupture (Figure 2B). An emergency aneurysm ligation operation was performed, and the left lateral thigh pain and foot drop improved gradually after the operation. After four months, a follow-up CT showed left kidney scarring but no recurrence of the aneurysms (Figure 3).

\section{DISCUSSION}

The present case involved infective endocarditis and was complicated with respect to both diagnosis and treatment. Initial diagnosis was difficult because infective endocarditis shares important symptoms and signs with vasculitis, and the initial presentation of infective endocarditis often mimics vasculitis. Patients may have renal or liver failure and skin lesions. Common manifestations of infective endocarditis include vague general symptoms such as anorexia, weight loss, malaise and fever (1), which are also found frequently in patients with vasculitis. The renal involvement of polyarteritis nodosa (PAN) is the most common manifestation that overlaps with infective endocarditis (3-5). Cutaneous lesions, which are found in more than $40 \%$ of PAN patients, are another common overlapping manifestation $(4,6)$.
Various systemic manifestations also interfere with the differential diagnosis of infective endocarditis and vasculitis. In PAN, common symptoms include fever (21\%), myalgia (52\%), extrarenal involvement of vasculitis (40\%) and hematuria (87\%) (3). Our patient initially presented with general prodromal symptoms and migrating skin lesions, with the multiple pseudoaneurysms and renal infarction developing later. Vasculitis, such as PAN, was suspected rather than an infectious condition because the patient was previously healthy and had no predisposing factors for infective endocarditis.

An atypical form of mycotic aneurysm also complicated the initial diagnosis. First, multiple extracranial mycotic aneurysms are rare. The prevalence of infected aortic aneurysms is $0.7 \%$ to $1 \%$ of all surgically treated aortic aneurysms (7). There are no epidemiological reports of multiple extracranial mycotic aneurysms, and only a few cases have been reported (8). Second, the internal iliac artery is not a common site for a mycotic aneurysm; the femoral artery is the most common site (38\%), followed by the abdominal aorta (31\%) (9).

Another remarkable finding in the present case was the aggravation of the aneurysms despite adequate medical and surgical treatment, which is uncommon (10). A mycotic aneurysm is a life-threatening condition with high mortality (7); thus, correct diagnosis and proper surgical treatment are crucial. Therefore, it is important for clinicians to recognize that an aneurysm could recur or progress despite proper medical management of infective endocarditis. If there are signs of disease progression despite appropriate treatment, clinicians should promptly consider further evaluation, follow-up imaging and blood cultures. In addition, although four to six weeks of parenteral antimicrobial therapy is generally recommended for the treatment of mycotic aneurysm, longer durations of antimicrobial therapy should be considered if clinical signs or symptoms persist.

\section{CONCLUSION}

The possibility of underlying infectious disease in patients presenting with symptoms and signs of vasculitis, and vice versa, should always be considered. The clinician should sustain a high index of suspicion because the treatment of each disease differs significantly and misdiagnosis can be life threatening.

CONFLICT OF INTEREST: The authors have no conflicts of interest to report.

\section{REFERENCES}

1. Mylonakis E, Calderwood S. Infective endocarditis in adults. N Engl J Med 2001;345:1318-30.

2. Majumdar A, Chowdhary S, Ferreira M, et al. Renal pathological findings in infective endocarditis. Nephrol Dial Transplant 2000;15:1782-7.

3. El-Reshaid K, Kapoor M, El-Reshaid W, et al. The spectrum of renal disease associated with microscopic polyangiitis and classic polyarteritis nodosa in Kuwait. Nephrol Dial Transplant 1997;12:1874-82.

4. Lane S, Watts R, Shepstone L, Scott D. Primary systemic vasculitis: Clinical features and mortality. Qjm 2005;98:97-111.

5. Westman K, Bygren P, Olsson H, et al. Relapse rate, renal survival, and cancer morbidity in patients with Wegener's granulomatosis or microscopic polyangiitis with renal involvement. J Am Soc Nephrol 1998;9:842-52.

6. Borrie P. Cutaneous polyarteritis nodosa. Br J Dermatol 1972;87:87-95

7. Lee W, Mossop P, Little A, et al. Infected (mycotic) aneurysms: Spectrum of imaging appearances and management. Radiographics 2008;28:1853-68.

8. Kaufman S, White Jr R, Harrington D, et al. Protean manifestations of mycotic aneurysms. Am J Roentgenol 1978;131:1019-25.

9. Brown S, Busuttil R, Baker J, et al. Bacteriologic and surgical determinants of survival in patients with mycotic aneurysms. J Vasc Surg 1984;1:541-7.

10. Bamford J, Hodges J, Warlow C. Late rupture of a mycotic aneurysm after "cure" of bacterial endocarditis. J Neurol 1986;233:51-3. 


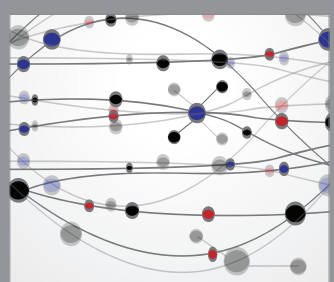

The Scientific World Journal
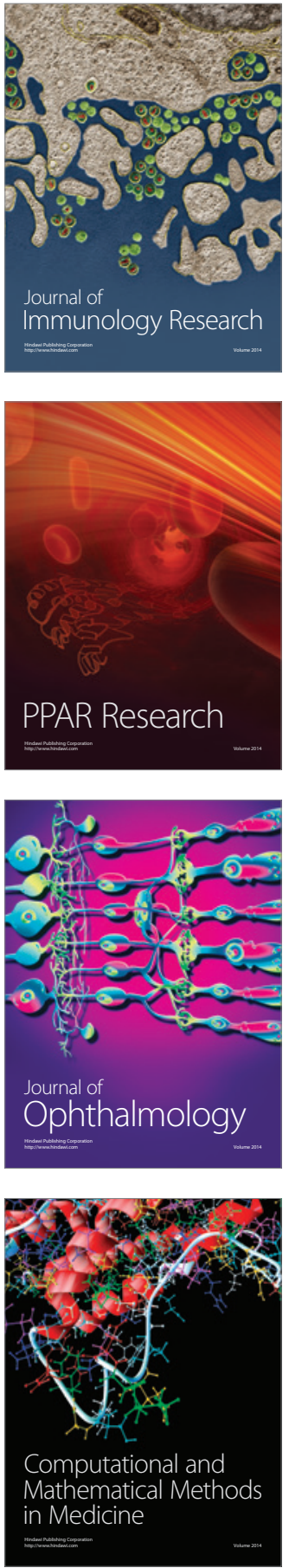

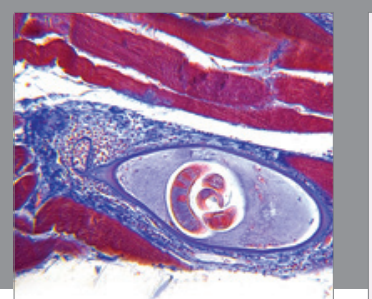

Gastroenterology Research and Practice

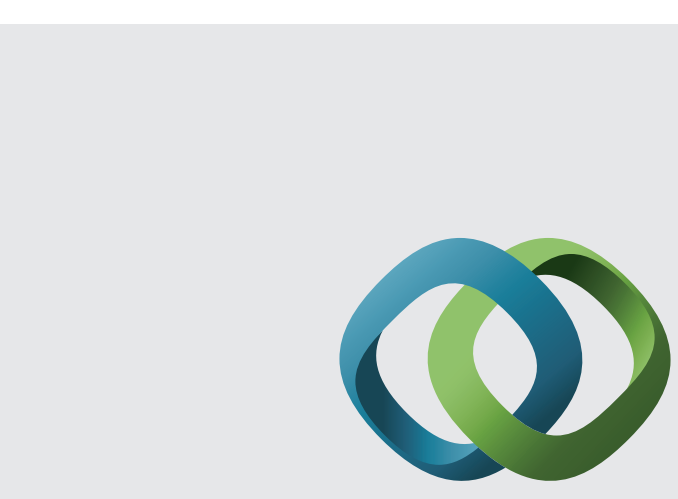

\section{Hindawi}

Submit your manuscripts at

http://www.hindawi.com
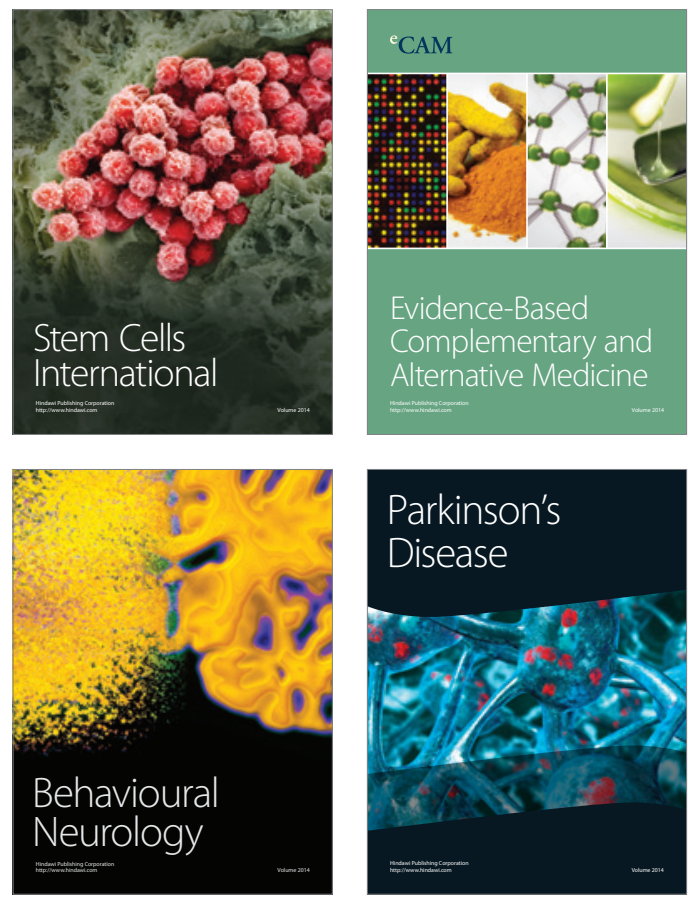
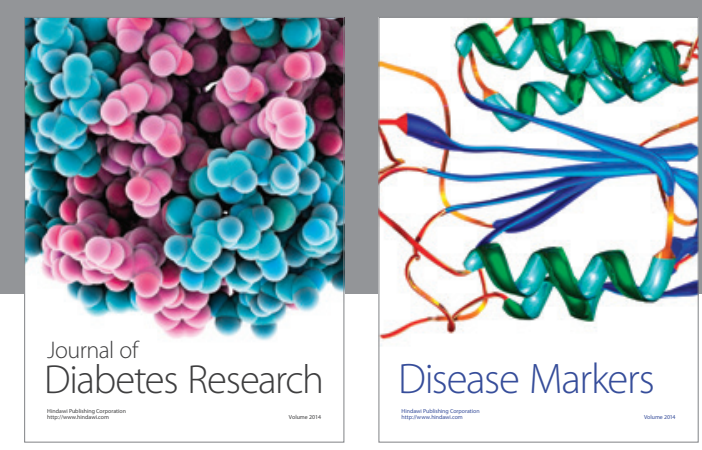

Disease Markers
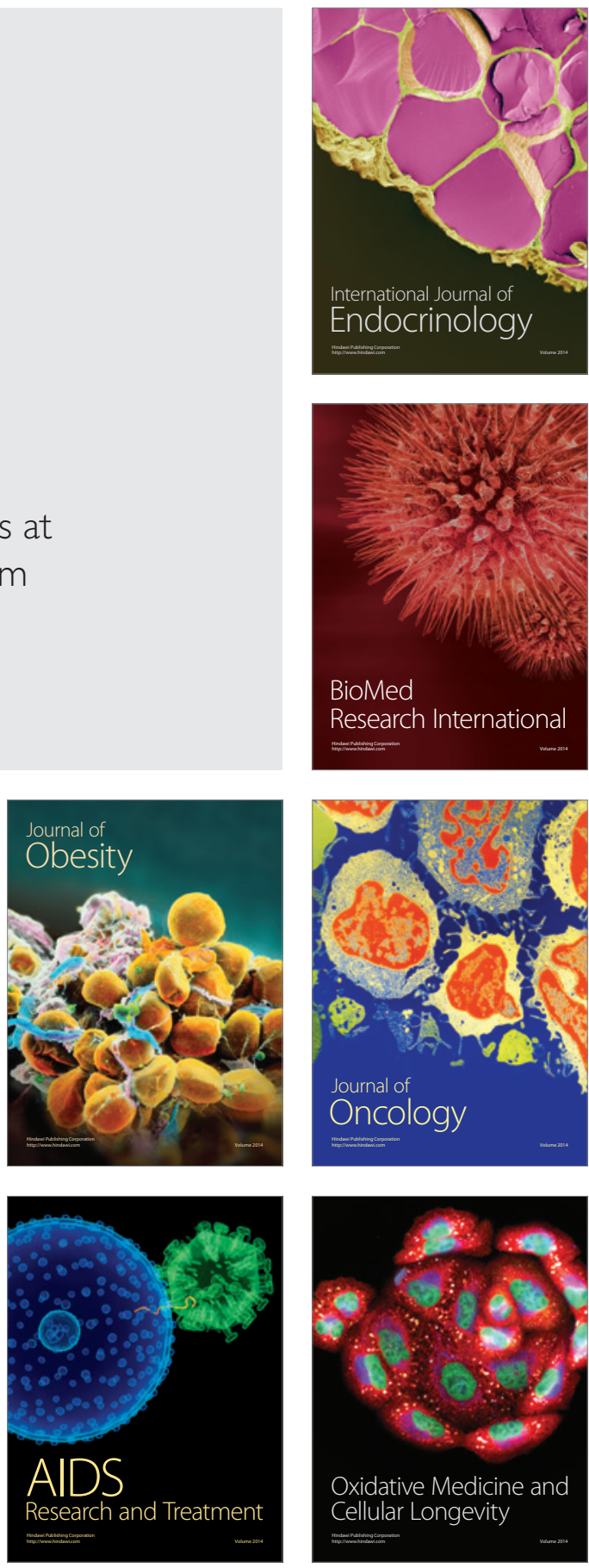